\title{
Classical gravitational self-energy from double copy
}

\author{
Gabriel Luz Almeida, ${ }^{a}$ Stefano Foffa $^{b}$ and Riccardo Sturani ${ }^{c}$ \\ ${ }^{a}$ Departamento de Física Teórica e Experimental, Universidade Federal do Rio Grande do Norte, \\ Av. Sen. Salgado Filho, Natal RN 59078-970, Brazil \\ ${ }^{b}$ Département de Physique Théorique and Center for Astroparticle Physics, Université de Genève, \\ 24 quai Ernest Ansermet, Geneva CH-1211, Switzerland \\ ${ }^{c}$ International Institute of Physics, Universidade Federal do Rio Grande do Norte, \\ Campus Universitário, Lagoa Nova CP:1613, Natal RN 59078-970, Brazil \\ E-mail: gabriel.luz@fisica.ufrn.br, stefano.foffa@unige.ch, \\ riccardo@iip.ufrn.br
}

ABSTRACT: We apply the classical double copy to the calculation of self-energy of composite systems with multipolar coupling to gravitational field, obtaining next-to-leading order results in the gravitational coupling $G_{N}$ by generalizing color to kinematics replacement rules known in literature. When applied to the multipolar description of the two-body system, the self-energy diagrams studied in this work correspond to tail processes, whose physical interpretation is of radiation being emitted by the non-relativistic source, scattered by the curvature generated by the binary system and then re-absorbed by the same source. These processes contribute to the conservative two-body dynamics and the present work represents a decisive step towards the systematic use of double copy within the multipolar post-Minkowskian expansion.

KEYwords: Classical Theories of Gravity, Effective Field Theories, Black Holes

ArXiv EPrint: 2008.06195 


\section{Contents}

1 Introduction 1

2 Method 2

3 Self-energy diagrams at leading order 4

3.1 Electric moments 5

3.2 Magnetic moments 6

4 Self-energy diagrams at next-to-leading order $\quad 8$

4.1 Tail diagrams with electric moments 8

$\begin{array}{ll}4.2 & \text { Tail diagrams with magnetic moments } \\ \end{array}$

5 Discussion 12

A Computation details $\quad 13$

A.1 Multipole expansion in dilaton-axion-gravity 13

$\begin{array}{ll}\text { A.2 Graviton, dilaton, axion action up to cubic interaction } & 15\end{array}$

$\begin{array}{ll}\text { B Vanishing integrals } & 15\end{array}$

\section{Introduction}

Links between the gauge and gravity theories first appeared in scattering amplitude computations, as first shown within a string theory context by the Kawai-Lewellen-Tye identities [1] relating tree level closed and open string amplitudes, later extended to a correspondence between $\mathrm{S}$ matrix elements in gauge theory and gravity [2]. More recently the Bern-Carrasco-Johansson (BCJ) formalism [3] has provided a general mechanism for viewing gravitons as double copies of gluons at perturbative level.

The BCJ relations state that squaring non-Abelian Yang-Mills amplitudes in generic dimension $d$, and applying a set of rules to map color into kinematics degrees of freedom, gravitational amplitudes are recovered, which however do not coincide with General Relativity but include a scalar, dilaton field and a 2-form gauge field $B_{\mu \nu}$. The BCJ double copy has been verified in a variety of supersymmetric field theories, see $[4,5]$ for reviews.

A remarkable application of double copy to non-perturbative classical solutions in Yang-Mills theory on one side, and Kerr-Schild black holes in General Relativity on the other, was shown in [6, 7], and further development on the classical side were made in [8], where the long-distance radiation gluon field emitted by a set of gauge charges has been computed and mapped into asymptotic radiation field in a theory of gravity plus a dilaton. This latter result has been extended in $[9,10]$ to the case of spinning particles, in [11] to next-to-leading order in coupling (in the post-Minkowskian regime of gravity) and in [12] to next-to-leading order with finite-size sources of non-zero spin, see also [13] for double copy application to gravitational radiation and spin effects. 
For other relevant work on the classical double copy: see [14] for application to the twobody effective gravitational potential in the post-Newtonian approximation, with possible problems arising at $O\left(G_{N}^{2}\right)$ with respect to leading order [15], and the seminal work [16, 17] for the determination of the two-body potential at third post-Minkowskian order.

In the present work we show the computation of self-energy diagrams representing forward scattering of non-relativistic sources described by their multipolar coupling to gauge and gravity fields to next-to-leading order in the gauge/gravity coupling, by extending previously derived rules for gauge charge/kinematic variable duality. According to standard post-Newtonian (PN) approximation to General Relativity [18], this processes contribute to the conservative two-body dynamics starting at $4 \mathrm{PN}$ order.

In the post-Newtonian approach to the two-body dynamics it is customary to separate the near from the far zone. In the former the interactions between the binary constituents are mediated by the constrained, non-radiative longitudinal modes of gravity, in the latter gravitational radiative degrees of freedom are also relevant and the source is modeled as a single object with multipoles. The real part of self-energy diagram amplitudes in the far zone complements the near zone derivation of the effective two-body dynamics [19, 20], while the imaginary part relates via the optical theorem to the radiated energy, following the now standard setup based on Non-Relativistic-General-Relativity (NRGR) [21].

The paper is structured as follows: in section 2 we introduce the double copy method applied to source coupled to gauge fields and gravity in the multipole expansion. In section 3 we give the details of the correspondence, verifying the matching of the "square" of the gauge self-energy amplitude with the General Relativity plus dilatonic and axionic amplitude, checking the correspondence at next-to-leading order in gauge/gravitational coupling in section 4 . We finally conclude in section 5 .

\section{Method}

We show how the mapping between the square of gauge amplitudes and gravity ones work in the case of multipole-expanded sources. On the gauge side we consider the bulk action ${ }^{1}$

$$
\mathcal{S}_{\text {bulk }}^{\text {(gauge) }}=-\int \mathrm{d}^{d+1} x\left[\frac{1}{4} F_{\mu \nu}^{a} F^{a \mu \nu}+\frac{1}{2}\left(\partial_{\mu} A^{a \mu}\right)^{2}\right]
$$

in terms of the field strength $F_{\mu \nu}^{a}$ with structure constant $f^{a b c}$, where we have displayed explicitly the Feynman gauge fixing term in terms of the gauge field $A_{\mu}^{a}$ (resulting in the standard propagator $P\left[A_{\mu}^{a}, A_{\nu}^{b}\right]=-i \delta^{a b} \eta_{\mu \nu} /\left(\mathbf{k}^{2}-k_{0}^{2}\right)$, boldface character denoting 3vectors), and a system of classical, spinning Yang-Mills color charges coupled to gluons, described by a trajectory $x^{\mu}$, a color charge $c_{a}$ and a spin $S^{\mu \nu}$ (all three depending on the world-line parameter $\tau$ ), whose dynamics is described by the world-line action summed over particles

$$
\begin{aligned}
\mathcal{S}_{\mathrm{wl}}^{\text {(gauge) }} & =\sum_{p \in \operatorname{parts}} g \int \mathrm{d} x^{\mu} c_{a p} A_{\mu}^{a}-\frac{\kappa}{2} \int \mathrm{d} \tau c_{a p} S_{p}^{\mu \nu} F_{\mu \nu}^{a} \\
& \simeq g \int \mathrm{d} t\left(q_{a} A_{0}^{a}+d_{a}^{i} F_{i 0}^{a}+\frac{1}{2} Q_{a}^{i j} F_{i 0, j}^{a}-\frac{1}{2}\left(\mu_{a k}+\kappa c_{a} S_{k}\right) \epsilon^{k i j} F_{i j}^{a}+\ldots\right),
\end{aligned}
$$

\footnotetext{
${ }^{1}$ We adopt the mostly plus signature for the metric, i.e. Minkowski metric $\eta_{\mu \nu}=\operatorname{diag}(-1,1, \ldots, 1)$.
} 
where in the second line we moved from the description in terms of fundamental constituents to the one in terms of an extended object with multipoles. ${ }^{2}$ The spin antisymmetric tensor $S^{\mu \nu}$ has 6 components, we then adopt a spin supplementary condition [22] to reduce them to the three physical ones, implying that $S^{i 0} \sim S^{i j} v^{j}$ (and $S^{k} \equiv \frac{1}{2} \epsilon^{k i j} S_{i j}$ in eq. (2.2)).

On the gravity side we have that the degrees of freedom are represented by the metric $g_{\mu \nu}$, the dilaton $\psi$ and the axion $B_{\mu \nu}$ with field strength $H_{\mu \nu \rho}$ defined by

$$
H_{\mu \nu \rho} \equiv \partial_{\mu} B_{\nu \rho}+\partial_{\rho} B_{\mu \nu}+\partial_{\nu} B_{\rho \mu}
$$

The gauge-fixed bulk action is ${ }^{3}$

$$
\mathcal{S}_{\text {bulk }}^{(g d a)}=\int \mathrm{d}^{d+1} x \sqrt{-g}\left[2 \Lambda^{2}\left(R-\frac{1}{2} \Gamma^{\mu} \Gamma_{\mu}\right)-2(d-1)(\partial \psi)^{2}-\frac{1}{6} e^{-\frac{4 \psi}{\Lambda}} H_{\mu \nu \rho} H^{\mu \nu \rho}-\left(\partial_{\mu} B^{\mu \nu}\right)^{2}\right],
$$

where $\Lambda \equiv\left(32 \pi G_{N}\right)^{-1 / 2}$ (it has dimension of $\sqrt{\text { mass/length }}$ in $d=3$ ) and $\Gamma^{\mu} \equiv \Gamma_{\nu \rho}^{\mu} g^{\nu \rho}$. The world-line action is

$$
\begin{aligned}
\mathcal{S}_{\mathrm{wl}}^{g d a}= & \sum_{p \in \text { parts }}-m_{p} \int_{p} \mathrm{~d} \tau e^{\frac{\psi}{\Lambda}}-\frac{1}{2} \int_{p} \mathrm{~d} \tau S^{\mu \nu} \Omega_{\mu \nu}+\frac{1}{4 \Lambda} \int_{p} \mathrm{~d} x^{\rho} H_{\rho \mu \nu} S_{p}^{\mu \nu} \\
\simeq & \int \mathrm{d} t\left\{\frac{1}{2} E h_{00}+\frac{1}{2} \epsilon^{i j k} L_{i} h_{0 j, k}+\frac{1}{2} I^{i j} \mathcal{E}_{i j}+\frac{1}{6} I^{i j k} \mathcal{E}_{i j, k}+\frac{2}{3} J^{i j} \mathcal{B}_{i j}+\ldots\right. \\
& \left.+\frac{1}{\Lambda}\left[-E \psi-\frac{1}{2}\left(I^{i j} \psi_{, i j}-I \ddot{\psi}\right)+\ldots+\frac{1}{4} S^{i j} H_{0 i j}-\frac{1}{3} J^{i j} \epsilon_{i k l} H_{0 j k, l}+\ldots\right]\right\},
\end{aligned}
$$

where the world-line coupling (inclusive of the angular velocity tensor $\Omega_{\mu \nu}[22]$ ) have been expanded in multipoles for an ensemble of particles or equivalently for a finite size source with small internal velocities: $h_{\mu \nu} \equiv g_{\mu \nu}-\eta_{\mu \nu}$ is the gravitational perturbation around the Minkowski metric $\eta_{\mu \nu}, E$ is the total energy of the source, $L^{i} \equiv \epsilon^{i j k} S_{j k}$ its total angular momentum (dual to the spin anti-symmetric tensor, whose mixed timespace polarization $S^{0 i}$ vanish in the source center of mass), $I^{i j}$ and $I^{i j k}$ are respectively the traceless mass quadrupole and octupole, $J^{i j}$ the magnetic quadrupole $J^{i j} \equiv$ $\frac{1}{2} \int \mathrm{d}^{d} x x^{k} T^{0 l}\left(\epsilon^{i k l} x^{j}+\epsilon^{j k l} x^{i}\right)$, and the eletric $\mathcal{E}_{i j}$ and magnetic $\mathcal{B}_{i j}$ parts of the Riemann tensor $R_{\nu \rho \sigma}^{\mu}$ in the source rest frame take, respectively, the form $\mathcal{E}_{i j}=R_{0 i 0 j}, \mathcal{B}_{i j}=$ $\frac{1}{2} \epsilon_{i k l} R_{0 j k l}$ (see appendix A for details).

By generalizing the double copy rules to the coupling of gluons to source multipole moments we will express the "square" of classical self-energy diagrams on the gauge side as classical self-energy diagrams in the gravitational theory, to $O\left(G_{N}\right)$ interactions beyond the leading order diagrams.

\footnotetext{
${ }^{2}$ Greek indices run over $d+1$ space-time dimensions, Latin indices $i, j \ldots$ over space coordinates only, Latin indices $a, b, \ldots, h$ run gauge color indices, $q^{a}$ is the gauge charge $q_{a} \equiv \sum_{p} c_{a}(\tau(t)), d_{a}^{i}$ the electric dipole $d_{a}^{i} \equiv \sum_{p} c_{a} x_{p}^{i}, Q_{a}^{i j} \equiv \sum_{p} c_{a} x_{p}^{i} x_{p}^{j}$ is the electric quadrupole, $\mu_{a k}$ the magnetic dipole $\mu_{k}^{a} \equiv \frac{1}{2} \epsilon_{k i j} \sum_{p} c_{a} \frac{d x_{p}^{i}}{d t} x_{p}^{j}$ and $\kappa$ is a numerical coefficient determining the strength of the chromomagnetic interaction.

${ }^{3}$ Note that the dilaton is not canonical normalized here, it (and $B_{\mu \nu}$ ) has canonical dimensions, as the metric fields $\phi, A_{i}, \sigma_{i j}$ that will be introduced in eq. (3.1).
} 


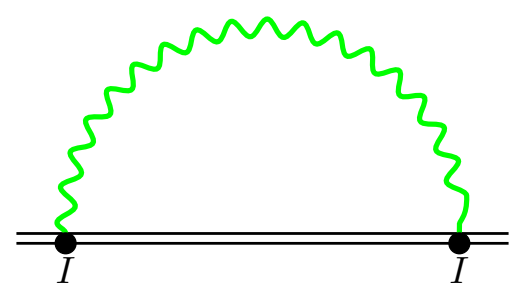

Figure 1. Self-energy diagram for a generic radiative multipole source $I$. The green wavy field represents the gauge/gravity interaction, while the black double line stands for the composite source.

\section{Self-energy diagrams at leading order}

We compute in this section self-energy diagrams like the one in figure 1, with generic multipole $I$ insertion at the extended object world-line.

We will compute quantities in space-dimension $d=3$ but it will be helpful to keep $d$ generic in the computations to check that explicit dependence on $d$ cancels in the sum of gravitational, dilatonic and axionic effective actions, as it happens in scattering amplitudes [23, 24].

In our non-relativistic setup we find convenient to use the Kaluza-Klein parametrization of the metric $[25]^{4}$

$$
g_{\mu \nu}=e^{2 \phi / \Lambda}\left(\begin{array}{cc}
-1 & \frac{A_{i}}{\Lambda} \\
\frac{A_{j}}{\Lambda} & e^{-c_{d} \phi / \Lambda}\left(\delta_{i j}+\frac{\sigma_{i j}}{\Lambda}\right)-\frac{A_{i} A_{j}}{\Lambda^{2}}
\end{array}\right),
$$

where $c_{d} \equiv 2(d-1) /(d-2)$ and $d$ is the number of purely space dimensions. Such decomposition has the virtue to provide diagonal propagators for the gravity fields, which we list below together with the dilaton and axion propagators

$$
\left.\begin{array}{rl}
P[\phi, \phi] & =-\frac{1}{2 c_{d}} \\
P\left[A_{i}, A_{j}\right] & =\frac{\delta_{i j}}{2} \\
P\left[\sigma_{i j}, \sigma_{k l}\right] & =-\frac{1}{2}\left(\delta_{i k} \delta_{j l}+\delta_{i l} \delta_{j k}-\frac{2}{d-2} \delta_{i j} \delta_{k l}\right) \\
P[\psi, \psi] & =-\frac{1}{4(d-1)} \\
P\left[B_{\mu \nu}, B_{\rho \sigma}\right] & =-\frac{1}{2}\left(\eta_{\mu \rho} \eta_{\nu \sigma}-\eta_{\mu \sigma} \eta_{\nu \rho}\right)
\end{array}\right\} \times \frac{i}{\mathbf{k}^{2}-k_{0}^{2}-i \epsilon} .
$$

The Lagrangian for the bulk fields up to cubic interactions is reported in eq. (A.9).

Below, we compute self-energy diagrams which, at leading order in $G_{N}(g)$, are due to processes represented by the diagram in figure 1. As it will be clear in the following, all such diagrams involve time derivatives of the source, hence the lowest non-vanishing

\footnotetext{
${ }^{4}$ We adopt the same symbol $A_{i}^{a}$ for the gauge field and $A_{i}$ for the mixed time-space component of gravity, the former being accompanied by the gauge index should avoid confusion between the two.
} 
on the gravity (gauge) side involves electric quadrupole (dipole) moments. The Green's function involved in the process is the Feynman one, which is the correct prescription for self-energy diagrams [26].

\subsection{Electric moments}

The lowest order non-vanishing self-energy diagram involve two quadrupole sources and in the gravity side receives contributions from exchange of gravitational and dilatonic modes: ${ }^{5}$

$$
\begin{gathered}
\mathcal{S}_{\mathrm{GR}}^{\left(I^{2}\right)}=-\frac{1}{8 \Lambda^{2}} \int \frac{\mathrm{d} k_{0}}{2 \pi} I^{i j}\left(k_{0}\right) I^{k l}\left(-k_{0}\right) \int_{\mathbf{k}} \frac{1}{\mathbf{k}^{2}-k_{0}^{2}}\left\{-\frac{1}{8} k_{0}^{4}\left(\delta_{i k} \delta_{j l}+\delta_{i l} \delta_{j k}-\frac{2}{d-2} \delta_{i j} \delta_{k l}\right)\right. \\
\left.+\frac{k_{0}^{2}}{4}\left(k_{i} k_{k} \delta_{j l}+k_{j} k_{l} \delta_{i k}\right)-\frac{1}{2 c_{d}}\left(k_{i} k_{j}+\frac{k_{0}^{2} \delta_{i j}}{d-2}\right)\left(k_{k} k_{l}+\frac{k_{0}^{2} \delta_{k l}}{d-2}\right)\right\}, \\
\mathcal{S}_{\psi}^{\left(I^{2}\right)}=\frac{1}{32(d-1) \Lambda^{2}} \int \frac{\mathrm{d} k_{0}}{2 \pi} I^{i j}\left(k_{0}\right) I^{k l}\left(-k_{0}\right) \int_{\mathbf{k}} \frac{1}{\mathbf{k}^{2}-k_{0}^{2}}\left(k_{i} k_{j}-k_{0}^{2} \delta_{i j}\right)\left(k_{k} k_{l}-k_{0}^{2} \delta_{k l}\right),
\end{gathered}
$$

and no contribution from the axion, as it does not couple to electric moments. Notably the sum of gravitational (3.3) and dilatonic (3.4) amplitudes display a factorizable structure, where terms explicitly dependent on the number of space dimensions $d$ cancel

$$
\mathcal{S}_{\mathrm{GR}+\psi}^{\left(I^{2}\right)}=\frac{1}{32 \Lambda^{2}} \int \frac{\mathrm{d} k_{0}}{2 \pi} I^{i j}\left(k_{0}\right) I^{k l}\left(-k_{0}\right) \int_{\mathbf{k}} \frac{1}{\mathbf{k}^{2}-k_{0}^{2}}\left(k_{i} k_{k}-k_{0}^{2} \delta_{i k}\right)\left(k_{j} k_{l}-k_{0}^{2} \delta_{j l}\right) .
$$

On the gauge side the electric dipole self-energy process gives

$$
\mathcal{S}_{A}^{\left(d^{2}\right)}=\frac{g^{2}}{2} \int \frac{\mathrm{d} k_{0}}{2 \pi} d^{a, i}\left(k_{0}\right) d^{b, k}\left(-k_{0}\right) \int_{\mathbf{k}} \frac{1}{\mathbf{k}^{2}-k_{0}^{2}}\left\langle F_{\mathbf{k} 0 i}^{a} F_{-\mathbf{k} 0 k}^{b}\right\rangle^{\prime}
$$

where primed brackets $\langle\cdots\rangle^{\prime}$ stands for field Green's functions stripped of factors $-i /\left(\mathbf{k}^{2}-\right.$ $\left.k_{0}^{2}\right)$ and delta functions for each propagator, e.g. $\int_{\mathbf{q}}\left\langle A_{\mathbf{k} \mu}^{a} A_{\mathbf{q} \nu}^{b}\right\rangle=-i /\left(\mathbf{k}^{2}-k_{0}^{2}\right)\left\langle A_{\mathbf{k} \mu}^{a} A_{-\mathbf{k} \nu}^{b}\right\rangle^{\prime}$.

Following standard procedure, we apply the substitutions ${ }^{6} g \rightarrow 1 /(2 \Lambda)$ and promote the gauge color indices to space index to "square" the integrand of eq. (3.6) according to the rule

$$
\begin{aligned}
d^{a, i} & \rightarrow \frac{I^{i j}}{2}, \\
\left\langle F_{\mathbf{k} 0 i}^{a} F_{-\mathbf{k} 0 k}^{b}\right\rangle^{\prime} & =\left(k_{0}^{2} \delta_{i k}-k_{i} k_{k}\right) \delta^{a b} \rightarrow\left(k_{0}^{2} \delta_{i k}-k_{i} k_{k}\right)\left(k_{0}^{2} \delta_{j l}-k_{j} k_{l}\right) .
\end{aligned}
$$

One then obtains

$$
\mathcal{S}_{A}^{\left(d^{2}\right)} \rightarrow \mathcal{S}_{\mathrm{DC}}^{\left(I^{2}\right)}=\frac{1}{32 \Lambda^{2}} \int \frac{\mathrm{d} k_{0}}{2 \pi} I^{i j}\left(k_{0}\right) I^{k l}\left(-k_{0}\right) \int_{\mathbf{k}} \frac{1}{\mathbf{k}^{2}-k_{0}^{2}}\left(k_{0}^{2} \delta_{i k}-k_{i} k_{k}\right)\left(k_{0}^{2} \delta_{j l}-k_{j} k_{l}\right),
$$

which equals the sum of eqs. (3.3) and (3.4) given in eq. (3.5).

\footnotetext{
${ }^{5}$ We adopt the notation $\int_{\mathbf{k}} \equiv \int \frac{\mathrm{d}^{d} k}{(2 \pi)^{d}}$.

${ }^{6}$ The $g \rightarrow 1 /(2 \Lambda)$ agrees with eq. (58) of [8], where $d$ denotes the number of space-time dimensions.
} 
The above results can be straightforwardly generalized to higher order $2^{r+2}$-th electric moments $I^{i j i_{1} \ldots i_{r}}$ for gravity

$$
\begin{aligned}
\mathcal{S}_{\mathrm{GR}}^{\left(I_{r+2}^{2}\right)}= & -\frac{1}{2[(r+2) !]^{2} \Lambda^{2}} \int \frac{\mathrm{d} k_{0}}{2 \pi} I^{i j i_{1} \cdots i_{r}}\left(k_{0}\right) I^{k l k_{1} \cdots k_{r}}\left(-k_{0}\right) \int_{\mathbf{k}} \frac{k_{i_{1}} \cdots k_{i_{r}} k_{k_{1}} \cdots k_{k_{r}}}{\mathbf{k}^{2}-k_{0}^{2}} \\
\times & \left\{-\frac{1}{8} k_{0}^{4}\left(\delta_{i k} \delta_{j l}+\delta_{i l} \delta_{j k}-\frac{2}{d-2} \delta_{i j} \delta_{k l}\right)\right. \\
& \left.+\frac{k_{0}^{2}}{4}\left(k_{i} k_{k} \delta_{j l}+k_{j} k_{l} \delta_{i k}\right)-\frac{1}{2 c_{d}}\left(k_{i} k_{j}+\frac{k_{0}^{2} \delta_{i j}}{d-2}\right)\left(k_{k} k_{l}+\frac{k_{0}^{2} \delta_{k l}}{d-2}\right)\right\},
\end{aligned}
$$

for the dilaton

$$
\begin{aligned}
\mathcal{S}_{\psi}^{\left(I_{r+2}^{2}\right)}= & \frac{1}{8(d-1)[(r+2) !]^{2} \Lambda^{2}} \int \frac{\mathrm{d} k_{0}}{2 \pi} I^{i j i_{1} \cdots i_{r}}\left(k_{0}\right) I^{k l k_{1} \cdots k_{r}}\left(-k_{0}\right) \\
& \times \int_{\mathbf{k}} \frac{k_{i_{1}} \cdots k_{i_{r}} k_{k_{1}} \cdots k_{k_{r}}}{\mathbf{k}^{2}-k_{0}^{2}}\left(k_{i} k_{j}-k_{0}^{2} \delta_{i j}\right)\left(k_{k} k_{l}-k_{0}^{2} \delta_{k l}\right),
\end{aligned}
$$

and for the gauge field coupling to the $2^{r+1}$ multipole $d^{a, i i_{1} \cdots i_{r}}$

$$
\mathcal{S}_{A}^{\left(d_{r+1}^{2}\right)}=\frac{g^{2}}{2[(r+1) !]^{2}} \int \frac{\mathrm{d} k_{0}}{2 \pi} d^{a, i i_{1} \ldots i_{r}}\left(k_{0}\right) d^{b, k k_{1} \ldots k_{r}}\left(-k_{0}\right) \int_{\mathbf{k}} \frac{k_{i_{1}} \ldots k_{i_{r}} k_{k_{1}} \ldots k_{k_{r}}}{\mathbf{k}^{2}-k_{0}^{2}}\left(k_{0}^{2} \delta_{i k}-k_{i} k_{k}\right) \text {. }
$$

Applying previous rules (3.7) completed with

$$
d^{a, i i_{1} \ldots i_{r}} \rightarrow \frac{1}{(r+2)} I^{i j i_{1} \ldots i_{r}}
$$

the double copy of the gauge electric dipole self-energy can be derived to be

$$
\begin{aligned}
& \mathcal{S}_{\mathrm{DC}}^{\left(I_{r+2}^{2}\right)}=\frac{1}{8[(r+2) !]^{2} \Lambda^{2}} \int \frac{\mathrm{d} k_{0}}{2 \pi} I^{i j i_{1} \ldots i_{r}}\left(k_{0}\right) I^{k l k_{1} \ldots k_{r}}\left(-k_{0}\right) \int_{\mathbf{k}} \frac{k_{i_{1}} \ldots k_{i_{r}} k_{k_{1}} \ldots k_{k_{r}}}{\mathbf{k}^{2}-k_{0}^{2}} \\
& \times\left(k_{i} k_{k}-k_{0}^{2} \delta_{i k}\right)\left(k_{j} k_{l}-k_{0}^{2} \delta_{j l}\right),
\end{aligned}
$$

which much like in the electric quadrupole case (3.8) equates the sum of (3.9) and (3.10).

\subsection{Magnetic moments}

In the magnetic multipole moment case we have from GR

$$
\mathcal{S}_{\mathrm{GR}}^{\left(J^{2}\right)}=\frac{\epsilon_{i m n} \epsilon_{k r s}}{36 \Lambda^{2}} \int \frac{\mathrm{d} k_{0}}{2 \pi} J^{i j}\left(k_{0}\right) J^{k l}\left(-k_{0}\right) \int_{\mathbf{k}} \frac{k_{n} k_{s}}{\mathbf{k}^{2}-k_{0}^{2}}\left[k_{0}^{2}\left(\delta_{j l} \delta_{m r}+\delta_{j r} \delta_{l m}\right)-k_{j} k_{l} \delta_{m r}\right],
$$

and from the axion

$$
\mathcal{S}_{B}^{\left(J^{2}\right)}=\frac{\epsilon_{i m n} \epsilon_{k r s}}{36 \Lambda^{2}} \int \frac{\mathrm{d} k_{0}}{2 \pi} J^{i j}\left(k_{0}\right) J^{k l}\left(-k_{0}\right) \int_{\mathbf{k}} \frac{k_{n} k_{s}}{\mathbf{k}^{2}-k_{0}^{2}}\left[k_{0}^{2}\left(\delta_{j l} \delta_{m r}-\delta_{j r} \delta_{l m}\right)-k_{j} k_{l} \delta_{m r}\right],
$$

with vanishing contribution from the dilaton. Note that in the sum of the gravitational and axionic contributions the terms where the Levi-Civita tensors have no indices contracted between themselves cancel, whereas the remaining ones add up. 
On the gauge side

$$
\mathcal{S}_{A}^{\left(\mu^{2}\right)}=\frac{g^{2}}{8} \epsilon_{i m n} \epsilon_{k r s} \int \frac{\mathrm{d} k_{0}}{2 \pi} \mu^{a i}\left(k_{0}\right) \mu^{b k}\left(-k_{0}\right) \int_{\mathbf{k}} \frac{1}{\mathbf{k}^{2}-k_{0}^{2}}\left\langle F_{\mathbf{k} m n}^{a} F_{-\mathbf{k} r s}^{b}\right\rangle^{\prime},
$$

and making the substitutions

$$
\begin{aligned}
\mu^{a i} & \rightarrow \frac{2}{3} J^{i j}, \\
\frac{1}{4} \epsilon_{i m n} \epsilon_{k r s}\left\langle F_{\mathbf{k} m n}^{a} F_{-\mathbf{k} r s}^{b}\right\rangle^{\prime} & =\epsilon_{i m n} \epsilon_{k r s} k_{m} k_{r} \delta_{n s} \delta^{a b} \\
& =\left(\mathbf{k}^{2} \delta_{i k}-k_{i} k_{k}\right) \delta^{a b} \rightarrow\left(\mathbf{k}^{2} \delta_{i k}-k_{i} k_{k}\right)\left(k_{0}^{2} \delta_{j l}-k_{j} k_{l}\right),
\end{aligned}
$$

one has

$$
\mathcal{S}_{A}^{\mu^{2}} \rightarrow \mathcal{S}_{\mathrm{DC}}^{J^{2}}=\frac{1}{18 \Lambda^{2}} \int \frac{\mathrm{d} k_{0}}{2 \pi} J^{i j}\left(k_{0}\right) J^{k l}\left(-k_{0}\right) \int_{\mathbf{k}} \frac{1}{\mathbf{k}^{2}-k_{0}^{2}}\left(\mathbf{k}^{2} \delta_{i k}-k_{i} k_{k}\right)\left(k_{0}^{2} \delta_{j l}-k_{j} k_{l}\right)
$$

which equates the sum of eqs. (3.14) and (3.15). Note that to obtain the gravitational magnetic result we did not "square" the gauge magnetic dipole result (3.16) but rather combine it with the electric dipole (3.6), which, beside being justified a posteriori as it gives the expected result, is the correct prescription for preserving magnetic parity.

Like for the self-energy electric dipole of subsection 3.1, this result can be generalized to all magnetic multipole moments $J^{i j i_{1} \ldots i_{r}}$, for standard gravity

$$
\begin{aligned}
\mathcal{S}_{\mathrm{GR}}^{\left(J_{r+2}^{2}\right)}= & \frac{1}{9[(r+2) !]^{2} \Lambda^{2}} \int \frac{\mathrm{d} k_{0}}{2 \pi} J^{i j i_{1} \ldots i_{r}}\left(k_{0}\right) J^{k l k_{1} \cdots k_{r}}\left(-k_{0}\right) \int_{\mathbf{k}} \frac{k_{i_{1}} \cdots k_{i_{r}} k_{k_{1}} \ldots k_{k_{r}}}{\mathbf{k}^{2}-k_{0}^{2}} \\
& {\left[\left(\delta_{i k} \mathbf{k}^{2}-k_{i} k_{k}\right)\left(\delta_{j l} k_{0}^{2}-k_{j} k_{l}\right)-k_{0}^{2} \epsilon_{i k n} \epsilon_{j l s} k_{n} k_{s}\right], }
\end{aligned}
$$

for the axion

$$
\begin{aligned}
\mathcal{S}_{B}^{\left(J_{r+2}^{2}\right)}= & \frac{1}{9[(r+2) !]^{2} \Lambda^{2}} \int \frac{\mathrm{d} k_{0}}{2 \pi} J^{i j i_{1} \cdots i_{r}}\left(k_{0}\right) J^{k l k_{1} \cdots k_{r}}\left(-k_{0}\right) \frac{k_{i_{1}} \cdots k_{i_{r}} k_{k_{1}} \cdots k_{k_{r}}}{\mathbf{k}^{2}-k_{0}^{2}} \\
& \times\left[\left(\delta_{i k} \mathbf{k}^{2}-k_{i} k_{k}\right)\left(\delta_{j l} k_{0}^{2}-k_{j} k_{l}\right)+k_{0}^{2} \epsilon_{i k n} \epsilon_{j l s} k_{n} k_{s}\right]
\end{aligned}
$$

and vanishing contribution from the dilaton.

On the gauge side one has

$$
\mathcal{S}_{A}^{\left(\mu_{r+1}^{2}\right)}=\frac{g^{2} \epsilon_{i m n} \epsilon_{k r s}}{8[(r+1) !]^{2}} \int \frac{\mathrm{d} k_{0}}{2 \pi} \mu^{a, i i_{1} \cdots i_{r}} \mu^{b, k k_{1} \cdots k_{r}} \int_{\mathbf{k}} \frac{k_{i_{1}} \cdots k_{i_{r}} k_{k_{1}} \cdots k_{k_{r}}}{\mathbf{k}^{2}-k_{0}^{2}}\left(\mathbf{k}^{2} \delta_{i k}-k_{i} k_{k}\right) \delta^{a b},
$$

which using the double copy rules (3.7) and (3.17), complemented with

$$
\mu^{a, i i_{1} \ldots i_{r}} \rightarrow \frac{4}{3(r+2)} J^{i j i_{1} \cdots i_{r}}
$$

one obtains

$$
\begin{aligned}
\mathcal{S}_{\mathrm{DC}}^{\left(J_{r+2}^{2}\right)}= & \frac{2}{9[(r+2) !]^{2} \Lambda^{2}} \int \frac{\mathrm{d} k_{0}}{2 \pi} J^{i j i_{1} \ldots i_{r}}\left(k_{0}\right) J^{k l j_{1} \ldots j_{r}}\left(-k_{0}\right) \\
& \times \int_{\mathbf{k}} \frac{k_{i_{1}} \ldots k_{i_{r}} k_{k_{1}} \ldots k_{k_{r}}}{\mathbf{k}^{2}-k_{0}^{2}}\left(\mathbf{k}^{2} \delta_{i k}-k_{i} k_{k}\right)\left(k_{0}^{2} \delta_{j l}-k_{j} k_{l}\right),
\end{aligned}
$$

equalling the sum of (3.19) and (3.20) much like in the magnetic quadrupole case (3.18). 


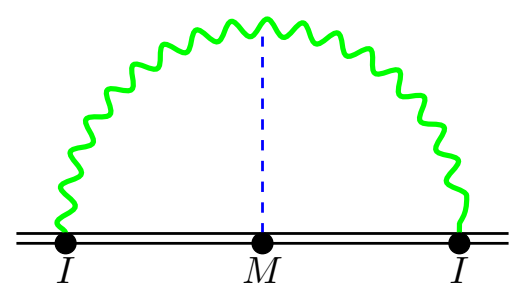

Figure 2. Self-energy diagram of the next-to-leading order process involving the scattering of modes emitted by a radiative multipole source $I$ onto a mode generated by a generic multipole $M$.

\section{Self-energy diagrams at next-to-leading order}

Having succeeded in the warm-up exercise of double-copying the self-energy processes without bulk interaction, we now move to the less trivial self-energy at next order in $G_{N}$ order, i.e. $O\left(G_{N}^{2}\right)$ which involves one cubic interaction in the bulk, see figure 2. One can distinguish two cases according to which type of source the third gravitational mode is attached to: a conserved multipole (like energy and angular momentum) or a radiative multipole. We will treat here the former case in which the additional world-line insertion has a total energy $E$ vertex, also known as tail process [27], as the back-scattering induced by the gravitational longitudinal mode sourced by the total energy induces radiation "tails" propagating inside the light cone.

\subsection{Tail diagrams with electric moments}

The pure gravitational process involving electric quadrupoles gives an effective action [28]

$$
\begin{aligned}
\mathcal{S}_{\mathrm{GR}}^{\left(E I^{2}\right)}= & \frac{E}{16 \Lambda^{4}} \int \frac{\mathrm{d} k_{0}}{2 \pi} I^{i j}\left(k_{0}\right) I^{k l}\left(-k_{0}\right) \int_{\mathbf{k}, \mathbf{q}} \frac{1}{\mathbf{k}^{2}-k_{0}^{2}} \frac{1}{(\mathbf{k}+\mathbf{q})^{2}-k_{0}^{2}} \frac{1}{\mathbf{q}^{2}} \\
\times & \left\{\frac{k_{0}^{6}}{8}\left(\delta_{i k} \delta_{j l}+\delta_{i l} \delta_{j k}-\frac{2 \delta_{i j} \delta_{k l}}{d-1}\right)\right. \\
& +\frac{k_{0}^{4}}{2}\left(2 \delta_{j l} k_{i} q_{k}+\frac{1}{2(d-1)}\left\{\left[(k+q)_{k}(k+q)_{l}+2 q_{k} q_{l}\right] \delta_{i j}+\delta_{k l} k_{i} k_{j}\right\}\right) \\
& +\frac{k_{0}^{2}}{2}\left(\frac{(d-2)}{2(d-1)} k_{i} k_{j}\left[(k+q)_{k}(k+q)_{l}+2 q_{k} q_{l}\right]\right. \\
& \left.\left.+k_{j}(k+q)_{l}\left[k_{k} q_{i}-k_{i} q_{k}-\mathbf{k} \cdot(\mathbf{k}+\mathbf{q}) \delta_{i k}\right]\right)\right\},
\end{aligned}
$$

and the dilaton contribution

$$
\begin{aligned}
\mathcal{S}_{\psi}^{\left(E I^{2}\right)}= & \frac{E}{64(d-1) \Lambda^{4}} \int \frac{\mathrm{d} k_{0}}{2 \pi} I^{i j}\left(k_{0}\right) I^{k l}\left(-k_{0}\right) \int_{\mathbf{k}, \mathbf{q}} \frac{1}{\mathbf{k}^{2}-k_{0}^{2}} \frac{1}{(\mathbf{k}+\mathbf{q})^{2}-k_{0}^{2}} \frac{1}{\mathbf{q}^{2}} \\
& \times\left\{k_{0}^{6} \delta_{i j} \delta_{k l}-k_{0}^{4}\left\{\left[(k+q)_{k}(k+q)_{l}+2 q_{k} q_{l}\right] \delta_{i j}+\delta_{k l} k_{i} k_{j}\right\}\right. \\
& \left.\quad+k_{0}^{2} k_{i} k_{j}\left((k+q)_{k}(k+q)_{l}+2 q_{k} q_{l}\right)\right\},
\end{aligned}
$$


with no contribution from the axion. The sum of the integrands of the gravitational and dilatonic contributions, eqs. (4.1) and (4.2), turns out to be independent of $d$ and can be recast in a "perfect square" form with the addition of (non-trivially) vanishing terms

$$
\begin{aligned}
\mathcal{S}_{\mathrm{GR}+\psi}^{\left(E I^{2}\right)}= & \frac{E}{64 \Lambda^{4}} \int \frac{\mathrm{d} k_{0}}{2 \pi} I^{i j}\left(k_{0}\right) I^{k l}\left(-k_{0}\right) \int_{\mathbf{k}, \mathbf{q}} \frac{1}{\mathbf{k}^{2}-k_{0}^{2}} \frac{1}{(\mathbf{k}+\mathbf{q})^{2}-k_{0}^{2}} \frac{1}{\mathbf{q}^{2}} \\
\times & k_{0}^{2}\left\{\left(k_{0}^{2} \delta_{i k}-(k+q)_{i} k_{k}-q_{i} q_{k}\right)\left(k_{0}^{2} \delta_{j l}-(k+q)_{j} k_{l}-q_{j} q_{l}\right)\right. \\
& +\delta_{j l}\left[2 k_{0}^{2}\left(q_{i} k_{k}+q_{k}(k+q)_{i}\right)-k_{i}(k+q)_{k}\left(\left(\mathbf{k}^{2}-k_{0}^{2}\right)+\left((\mathbf{k}+\mathbf{q})^{2}-k_{0}^{2}\right)-\mathbf{q}^{2}\right)\right] \\
& \left.+\left[k_{i} k_{j} q_{k} q_{l}-q_{i} q_{j}(k+q)_{k}(k+q)_{l}\right]\right\},
\end{aligned}
$$

which is suggestive of the double-copy structure, as the last two lines of eq. (4.3) can be shown to identically vanish, see appendix B for details.

The analog process on the gauge side, i.e. electric dipole self-energy at $O\left(g^{2}\right)$ with respect to leading order with conserved charge insertion, contributes to the effective action according to

$$
\begin{aligned}
\mathcal{S}_{A}^{\left(q d^{2}\right)}= & i \frac{g^{4}}{2} q^{a} \int \frac{\mathrm{d} k_{0}}{2 \pi} d_{i}^{b}\left(k_{0}\right) d_{k}^{c}\left(-k_{0}\right)\left(i f^{d e f}\right) \int_{\mathbf{k}, \mathbf{q}} \frac{1}{\mathbf{k}^{2}-k_{0}^{2}} \frac{1}{(\mathbf{k}+\mathbf{q})^{2}-k_{0}^{2}} \frac{1}{\mathbf{q}^{2}} \\
& \left\langle\left(A_{0}^{a} F_{\mathbf{k} 0 i}^{b} F_{-\mathbf{k}-\mathbf{q} 0 k}^{c}\right)\left(\partial_{\mu} A_{\nu}^{d} A_{\rho}^{e} A_{\sigma}^{f}\right)\right\rangle^{\prime} \eta^{\mu \rho} \eta^{\mu \sigma} \\
= & -g^{4} q^{a} \int \frac{\mathrm{d} k_{0}}{2 \pi} d_{i}^{b}\left(k_{0}\right) d_{k}^{c}\left(-k_{0}\right)\left(i f^{a b c}\right) \int_{\mathbf{k}, \mathbf{q}} \frac{1}{\mathbf{k}^{2}-k_{0}^{2}} \frac{1}{(\mathbf{k}+\mathbf{q})^{2}-k_{0}^{2}} \frac{1}{\mathbf{q}^{2}} \\
& \times k_{0}\left[k_{0}^{2} \delta_{i k}-(k+q)_{i} k_{k}-q_{i} q_{k}\right],
\end{aligned}
$$

where in the first passage Green's functions are not to be taken between fields within the same parenthesis and we adopted a mixed direct-Fourier space notation.

Using the gauge-gravity mapping rules derived in the previous section, completed with [3]

$$
i f^{a b c} \rightarrow \Gamma^{\mu \nu \rho}\left(k_{1}, k_{2}, k_{3}\right) \equiv \frac{1}{2}\left[\eta^{\mu \nu}\left(k_{1}-k_{2}\right)^{\rho}+\eta^{\rho \mu}\left(k_{3}-k_{1}\right)^{\nu}+\eta^{\nu \rho}\left(k_{2}-k_{3}\right)^{\mu}\right],
$$

and

$$
\begin{aligned}
\left\langle F_{\mathbf{k} 0 i}^{a} A_{\mu}^{b}\right\rangle^{\prime} & =i\left(k_{0} \eta_{i \mu}-k_{i} \eta_{0 \mu}\right) \delta^{a b} \rightarrow-\left(k_{0} \eta_{i \mu}-k_{i} \eta_{0 \mu}\right)\left(k_{0} \eta_{k \mu^{\prime}}-k_{k} \eta_{0 \mu^{\prime}}\right) \\
\left\langle A_{0}^{a} A_{\mu}^{b}\right\rangle^{\prime} & =\eta_{0 \mu} \delta^{a b} \rightarrow \eta_{0 \mu} \eta_{0 \rho} \\
J^{a 0} & \rightarrow T^{00}, \quad q^{a} \rightarrow E
\end{aligned}
$$

eq. (4.4) can be double-copied into

$$
\begin{aligned}
\mathcal{S}_{\mathrm{DC}}^{\left(E I^{2}\right)}= & \frac{E}{64 \Lambda^{4}} \int \frac{\mathrm{d} k_{0}}{2 \pi} I^{i j}\left(k_{0}\right) I^{k l}\left(-k_{0}\right) \int_{\mathbf{k}, \mathbf{q}} \frac{1}{\mathbf{k}^{2}-k_{0}^{2}} \frac{1}{(\mathbf{k}+\mathbf{q})^{2}-k_{0}^{2}} \frac{1}{\mathbf{q}^{2}} \\
& \times k_{0}^{2}\left[k_{0}^{2} \delta_{i k}-(k+q)_{i} k_{k}-q_{i} q_{k}\right]\left[k_{0}^{2} \delta_{j l}-(k+q)_{j} k_{l}-q_{j} q_{l}\right],
\end{aligned}
$$


which exactly matches the sum of gravitational (4.1) and dilatonic (4.2) electric quadrupole tail self-energies at $O\left(G_{N}^{2}\right)$.

The hereditary (non-local in time) structure of this diagram comes from the terms $\sim k_{0}^{6} I_{i j}^{2}$, which displays a divergence from the integration region $\mathbf{q} \rightarrow 0, \mathbf{k} \rightarrow \infty$. After the $\mathbf{k}, \mathbf{q}$ integration a logarithmic piece of the type $\int \mathrm{d} k_{0} k_{0}^{6} \log \left(k_{0}\right) I_{i j}^{2}$ is obtained, that is local in $k_{0}$-space but translates in direct space into $\int_{-\infty}^{\infty} \mathrm{d} t I_{i j}(t) \int_{0}^{\infty} \frac{\mathrm{d} \tau}{\tau} I_{i j}^{(6)}(t-\tau)$ which is the long-known hereditary term $[27,28]$.

Generalization to higher order electric multipole moment is straightforward and gives for the gravity+dilaton process:

$$
\begin{aligned}
\mathcal{S}_{\mathrm{GR}+\psi}^{\left(E I_{r+2}^{2}\right)}= & \frac{E}{16[(r+2) !]^{2} \Lambda^{4}} \int \frac{\mathrm{d} k_{0}}{2 \pi} I^{i j i_{1} \cdots i_{r}}\left(k_{0}\right) I^{k l k_{1} \cdots k_{r}}\left(-k_{0}\right) \\
& \int_{\mathbf{k}, \mathbf{q}} \frac{k_{i_{1}} \cdots k_{i_{r}}}{\mathbf{k}^{2}-k_{0}^{2}} \frac{(k+q)_{k_{1}} \cdots(k+q)_{k_{r}}}{(\mathbf{k}+\mathbf{q})^{2}-k_{0}^{2}} \frac{1}{\mathbf{q}^{2}} \\
& \times k_{0}^{2}\left\{\left(k_{0}^{2} \delta_{i k}-(k+q)_{i} k_{k}-q_{i} q_{k}\right)\left(k_{0}^{2} \delta_{j l}-(k+q)_{j} k_{l}-q_{j} q_{l}\right)\right. \\
& +\delta_{j l}\left[2 k_{0}^{2}\left(q_{i} k_{k}+q_{k}(k+q)_{i}\right)-k_{i}(k+q)_{k}\left(\left(\mathbf{k}^{2}-k_{0}^{2}\right)+\left((\mathbf{k}+\mathbf{q})^{2}-k_{0}^{2}\right)-\mathbf{q}^{2}\right)\right] \\
& \left.+\left[k_{i} k_{j} q_{k} q_{l}-q_{i} q_{j}(k+q)_{k}(k+q)_{l}\right]\right\},
\end{aligned}
$$

which matches the double copy of the gauge amplitude

$$
\begin{aligned}
\mathcal{S}_{A}^{\left(q d_{r+1}^{2}\right)}=- & \frac{g^{4} q^{a}}{[(r+1) !]^{2}} \int \frac{\mathrm{d} k_{0}}{2 \pi} d^{b, i i_{1} \cdots i_{r}}\left(k_{0}\right) d^{c, k k_{1} \cdots k_{r}}\left(-k_{0}\right)\left(i f^{a b c}\right) \\
& \times \int_{\mathbf{k}, \mathbf{q}} \frac{1}{\mathbf{k}^{2}-k_{0}^{2}} \frac{1}{(\mathbf{k}+\mathbf{q})^{2}-k_{0}^{2}} \frac{1}{\mathbf{q}^{2}} \\
& \quad \times k_{i_{1}} \cdots k_{i_{r}}(k+q)_{k_{1}} \cdots(k+q)_{k_{r}} k_{0}\left[k_{0}^{2} \delta_{i k}-(k+q)_{i} k_{k}-q_{i} q_{k}\right],
\end{aligned}
$$

using the correspondence dictionary already established and the fact that the last two lines of eq. (4.8) vanish, as demonstrated in appendix B.

\subsection{Tail diagrams with magnetic moments}

Analogously, for the tail of the magnetic quadrupole one can compute the contribution to the self-energy at $O\left(G_{N}^{2}\right)$ from the purely gravitational sector (no-dilaton involved at any vertex) [26]

$$
\begin{aligned}
\mathcal{S}_{\mathrm{GR}}^{\left(E J^{2}\right)}= & \frac{E}{9 \Lambda^{4}} \int \frac{\mathrm{d} k_{0}}{2 \pi} J^{i j}\left(k_{0}\right) J^{k l}\left(-k_{0}\right) \int_{\mathbf{k}, \mathbf{q}} \frac{1}{\mathbf{k}^{2}-k_{0}^{2}} \frac{1}{(\mathbf{k}+\mathbf{q})^{2}-k_{0}^{2}} \frac{1}{\mathbf{q}^{2}} \times \\
& \left\{\frac{k_{0}^{4}}{8}\left[\delta_{j l}\left(\delta_{i k} \mathbf{k} \cdot(\mathbf{k}+\mathbf{q})-k_{k}(k+q)_{i}\right)+\epsilon_{i l r} \epsilon_{k j s} k^{r}(k+q)^{s}\right]\right. \\
& +\frac{k_{0}^{2}}{4} k_{j}\left[q_{l}\left(\delta_{i k} \mathbf{k} \cdot(\mathbf{k}+\mathbf{q})-k_{k}(k+q)_{i}\right)-\epsilon_{i l n} \epsilon_{k r s} k^{n} k^{r} q^{s}\right] \\
& \left.-\frac{1}{8} k_{j}(k+q)_{l}\left[\mathbf{k} \cdot(\mathbf{k}+\mathbf{q})\left(\delta_{i k} \mathbf{k} \cdot(\mathbf{k}+\mathbf{q})-k_{k}(k+q)_{i}\right)+\epsilon_{i m n} \epsilon_{k r s} k^{m} q^{n} k^{r} q^{s}\right]\right\},
\end{aligned}
$$


to which the axionic contribution only must be added. The axion couples to both the dilaton and the gravity field $\phi$ (coupling in the last two lines of eq. (A.9)), but the computation can be simplified by observing that the process involving a $\psi$ exactly cancels the process involving the Lagrangian where $\phi$ couples to $H_{\mu \nu \rho}^{2}$, with the only contribution coming from the coupling $\phi H_{i j 0}^{2}$, see eq. (A.9). In summary one gets

$$
\begin{aligned}
\mathcal{S}_{B}^{\left(E J^{2}\right)}= & \frac{E}{72 \Lambda^{4}} \int \frac{\mathrm{d} k_{0}}{2 \pi} J^{i j}\left(k_{0}\right) J^{k l}\left(-k_{0}\right) \int_{\mathbf{k}, \mathbf{q}} \frac{1}{\mathbf{k}^{2}-k_{0}^{2}} \frac{1}{(\mathbf{k}+\mathbf{q})^{2}-k_{0}^{2}} \frac{1}{\mathbf{q}^{2}} \\
& \times\left\{k_{0}^{4}\left[\delta_{j l}\left(\delta_{i k} \mathbf{k} \cdot(\mathbf{k}+\mathbf{q})-k_{k}(k+q)_{i}\right)-\epsilon_{i l r} \epsilon_{k j s} k^{r}(k+q)^{s}\right]\right. \\
& +k_{0}^{2}\left[-\left((k+q)_{j}(k+q)_{l}+k_{j} k_{l}\right)\left(\delta_{i k} \mathbf{k} \cdot(\mathbf{k}+\mathbf{q})-k_{k}(k+q)_{i}\right)+2 k_{j} \epsilon_{i l n} \epsilon_{k r s} k^{n} k^{r} q^{s}\right] \\
& \left.+k_{j}(k+q)_{l}\left[\mathbf{k} \cdot(\mathbf{k}+\mathbf{q})\left(\delta_{i k} \mathbf{k} \cdot(\mathbf{k}+\mathbf{q})-k_{k}(k+q)_{i}\right)+\epsilon_{i m n} \epsilon_{k r s} k^{m} q^{n} k^{r} q^{s}\right]\right\} .
\end{aligned}
$$

For the magnetic quadrupole, like in the leading order self-energy, all terms with the LeviCivita tensors cancel when adding the gravity and axionic contributions, to give

$$
\begin{aligned}
S_{\mathrm{GR}+B}^{\left(E J^{2}\right)}= & \frac{E}{36 \Lambda^{4}} \int \frac{\mathrm{d} k_{0}}{2 \pi} J^{i j}\left(k_{0}\right) J^{k l}\left(-k_{0}\right) \int_{\mathbf{k}, \mathbf{q}} \frac{1}{\mathbf{k}^{2}-k_{0}^{2}} \frac{1}{(\mathbf{k}+\mathbf{q})^{2}-k_{0}^{2}} \frac{1}{\mathbf{q}^{2}} \\
\times & \left\{k_{0}^{2}\left[\delta_{i k} \mathbf{k} \cdot(\mathbf{k}+\mathbf{q})-(k+q)_{i} k_{k}\right]\left[k_{0}^{2} \delta_{j l}-k_{l}(k+q)_{j}-q_{j} q_{l}\right]\right. \\
& \left.+\frac{k_{0}^{2}}{2}\left[\delta_{i k} \mathbf{k} \cdot(\mathbf{k}+\mathbf{q})-(k+q)_{i} k_{k}\right]\left[k_{j} q_{l}+q_{j}(k+q)_{l}\right]\right\},
\end{aligned}
$$

which indicates a factorizable structure, even though not a perfect square, by observing that the integral of the last line vanishes, see explicit computations in appendix B.

Computing the magnetic dipole tail diagram on the gauge side one gets

$$
\begin{aligned}
\mathcal{S}_{A}^{\left(q \mu^{2}\right)}= & i \frac{q^{a} g^{4}}{8} \int \frac{\mathrm{d} k_{0}}{2 \pi} \mu^{b i}\left(k_{0}\right) \mu^{c k}\left(-k_{0}\right)\left(i f^{d e f}\right) \epsilon_{i m n} \epsilon_{k r s} \\
& \times \int_{\mathbf{k}, \mathbf{q}} \frac{1}{\mathbf{k}^{2}-k_{0}^{2}} \frac{1}{\left(\mathbf{k}^{2}+\mathbf{q}^{2}\right)-k_{0}^{2}} \frac{1}{\mathbf{q}^{2}}\left\langle\left(A_{0}^{a} F_{\mathbf{k} m n}^{b} F_{-\mathbf{k}-\mathbf{q} r s}^{c}\right)\left(\partial_{\mu} A_{\nu}^{d} A_{\rho}^{e} A_{\sigma}^{f}\right)\right\rangle^{\prime} \eta^{\mu \rho} \eta^{\nu \sigma} \\
=- & q^{a} g^{4} \int \frac{\mathrm{d} k_{0}}{2 \pi} \mu^{b i}\left(k_{0}\right) \mu^{c k}\left(-k_{0}\right)\left(i f^{a b c}\right) \int_{\mathbf{k}, \mathbf{q}} \frac{1}{\mathbf{k}^{2}-k_{0}^{2}} \frac{1}{(\mathbf{k}+\mathbf{q})^{2}-k_{0}^{2}} \frac{1}{\mathbf{q}^{2}} \\
& \quad \times k_{0}\left(\delta_{i k} \mathbf{k} \cdot(\mathbf{k}+\mathbf{q})-k_{k}(k+q)_{i}\right) .
\end{aligned}
$$

Much like in the leading order self-energy for magnetic sources, to reproduce the gravitational plus axionic magnetic quadrupole tail one should not square (4.13), but rather combine it with the electric tail eq. (4.4), according to previously derived rules (3.7), (3.17) and (4.6) complemented with

$$
\frac{1}{2} \epsilon_{i m n}\left\langle F_{\mathbf{k} m n}^{a} A_{\mu}^{b}\right\rangle^{\prime}=i \epsilon_{i m \mu} k_{m} \delta^{a b} \rightarrow-\epsilon_{i m \mu} k_{m}\left(k_{j} \eta_{0 \nu}-k_{0} \eta_{j \nu}\right),
$$


which is consistent with replacing $\delta^{a b}$ with a contraction between a gauge field and an electric field $\sim\left\langle F_{\mathbf{k} j 0} A_{\nu}\right\rangle^{\prime}$. One then obtains

$$
\begin{aligned}
\mathcal{S}_{\mathrm{DC}}^{\left(E J^{2}\right)}= & \frac{E}{36 \Lambda^{4}} \int \frac{\mathrm{d} k_{0}}{2 \pi} J^{i j}\left(k_{0}\right) J^{k l}\left(-k_{0}\right) \int_{\mathbf{k}, \mathbf{q}} \frac{1}{\mathbf{k}-k_{0}^{2}} \frac{1}{(\mathbf{k}+\mathbf{q})^{2}-k_{0}^{2}} \frac{1}{\mathbf{q}^{2}} \times \\
& {\left[k_{0}^{2}\left(\delta_{i k} \mathbf{k} \cdot(\mathbf{k}+\mathbf{q})-k_{k}(k+q)_{i}\right)\left(k_{0}^{2} \delta_{j l}-k_{l}(k+q)_{j}-q_{j} q_{l}\right)\right], }
\end{aligned}
$$

which matches (4.12).

Finally we give the formula for the double copy in the case of higher order magnetic moments $J^{i j i_{1} \cdots i_{r}}$ for gravity+axion exchange:

$$
\begin{aligned}
\mathcal{S}_{\mathrm{DC}}^{\left(E J_{r+2}^{2}\right)}= & \frac{E}{9[(r+2) !]^{2} \Lambda^{4}} \int \frac{\mathrm{d} k_{0}}{2 \pi} J^{i j i_{1} \ldots i_{r}}\left(k_{0}\right) J^{k l k_{1} \ldots k_{r}}\left(-k_{0}\right) \\
& \int_{\mathbf{k}, \mathbf{q}} \frac{1}{\mathbf{k}^{2}-k_{0}^{2}} \frac{1}{(\mathbf{k}+\mathbf{q})^{2}-k_{0}^{2}} \frac{1}{\mathbf{q}^{2}} k_{i_{1}} \cdots k_{i_{r}}(k+q)_{k_{1}} \cdots(k+q)_{k_{r}} \\
& \times\left[k_{0}^{2}\left(\delta_{i k} \mathbf{k} \cdot(\mathbf{k}+\mathbf{q})-k_{k}(k+q)_{i}\right)\left(k_{0}^{2} \delta_{j l}-k_{l}(k+q)_{j}-q_{j} q_{l}\right)\right] .
\end{aligned}
$$

\section{Discussion}

The question of how the classical two-body gravitational potential may be extracted from quantum scattering amplitudes has a long history and investigation has been revived recently by works extending the class of double-copy applications to perturbative solutions of the equations of motions and to the effective action of a binary system. Our investigations aim at providing further evidence that the classical double copy can be applied to the derivation of gravitational two-body potential, which is relevant for theoretical modeling of gravitational wave sources.

In particular the post-Newtonian approach has been useful in constructing templates for gravitational wave data analysis and it decomposes the problem into a near and a far zone, the former involving longitudinal modes only, whereas the latter includes both longitudinal and radiative gravitational modes. Focusing on the far zone, where the gravitational wave source is defined as an extended object with multipoles, we have shown how the next-to-leading order in the Newton's constant responsible for tail terms in the effective potential can be reproduced with a double-copy procedure, applied for the first time to the multipolar post-Minkowskian expansion.

Classical bound states emitting radiation have been considered at leading order [29] and at next-to-leading order in [11], where, differently from our approach, the 2-body system is described in terms of individual binary constituent world-lines. A natural question that may arise is if the maps (3.12), (3.22) between multipoles in the gauge and gravity theory may be derived from the fundamental coupling of point particles. While one could expect so, we have been unable so far to establish such correspondence, which however remains a goal for the development of the specific double copy program started here.

Additional future applications to post-Newtonian, post-Minkowskian and multipolar approximations include application to $O\left(G_{N}^{2}\right)$ self-energy processes contributing to the 
effective action under the name of memory effect, and a systematization to higher order will be necessary. Note however that care is needed when comparing $G_{N}$ order among different approaches: in our case, for instance, we have terms $G_{N}^{2} \dddot{I}_{i j}^{2}$ that when expressed in terms of individual binary system constituent kinematic variables, involve acceleration and higher derivatives which can be expressed in terms of position and velocity via equations of motion. The tail term, studied in section 4, is e.g. a fourth post-Newtonian term giving rise to $G_{N}^{4}$ terms in the equations of motion.

\section{Acknowledgments}

The work of R.S. is partly supported by CNPq. The research of R.S. was partly supported by ICTP-SAIFR, by the International Centre for Theoretical Sciences (ICTS) during a visit for participating in the program - The Future of Gravitational-Wave Astronomy (Code: ICTS/fgwa2019/08) and by the Munich Institute for Astro- and Particle Physics (MIAPP) which is funded by the Deutsche Forschungsgemeinschaft (DFG, German Research Foundation) under Germany's Excellence Strategy-EXC-2094-390783311. G.L.A. thanks the research funding agency CAPES for the Ph.D. scholarship. S.F. is supported by the Fonds National Suisse and by the SwissMap NCCR.

\section{A Computation details}

\section{A.1 Multipole expansion in dilaton-axion-gravity}

The mutipole expansion is obtained by Taylor expanding the terms bilinear in the source and the gravitational-dilatonic-axionic field, and collecting terms at the same order of $v$, being $v$ the typical internal velocity of the source.

Describing the source as a continuous extended body (instead of the equivalent description of a collection of point particle used in section 2), one can characterize the source with its energy momentum tensor $T^{\mu \nu}$ and spin density $s^{\mu \nu}$ extended over a volume $V$ :

$$
\mathcal{S}_{\text {source }}=\int \mathrm{d} t \int_{V} \mathrm{~d}^{3} x\left(\frac{1}{2} T^{\mu \nu} h_{\mu \nu}+T \frac{\psi}{\Lambda}+\frac{1}{4 \Lambda} \dot{x}^{\rho} s^{\mu \nu} H_{\mu \nu \rho}\right) .
$$

Considering that the source is localized in a region or size $r$ much smaller than the radiation wavelength $\lambda_{r} \sim 2 \pi / \omega \sim r / v$ one can Taylor expand $\mathcal{S}_{\text {source }}$ to obtain

$$
\begin{aligned}
\mathcal{S}_{\text {mult }} \simeq & \int \mathrm{d} t\left\{\left(\int_{V} \mathrm{~d}^{3} x T^{00}\right) h_{00}+\left[2\left(\int_{V} \mathrm{~d}^{3} x T^{0 i}\right) h_{0 i}+\left(\int_{V} \mathrm{~d}^{3} x T^{00} x^{i}\right) h_{00, i}\right]\right. \\
+ & {\left[\left(\int_{V} \mathrm{~d}^{3} x T^{i j}\right) h_{i j}+\left(\int_{V} \mathrm{~d}^{3} x T^{0 i} x^{j}\right)\left(h_{0 i, j}+h_{0 j, i}\right)+\frac{1}{2}\left(\int_{V} \mathrm{~d}^{3} x T^{00} x^{i} x^{j}\right) h_{00, i j}\right] } \\
& \left.\left(\int_{V} \mathrm{~d}^{3} x T^{0 i} x^{j}\right)\left(h_{0 i, j}-h_{0 j, i}\right)+\left(\int_{V} \mathrm{~d}^{3} x T^{i j} x^{k}\right) h_{i j, k}+\ldots\right\} .
\end{aligned}
$$


Note that this Taylor expansion is actually an expansion in $r / \lambda_{r} \sim v$. Using repeatedly the energy-momentum conservation in the form $\dot{T}^{\mu 0}=-T_{, i}^{\mu i}$ one can derive

$$
\begin{aligned}
\int_{V} \mathrm{~d}^{3} x T^{i j} & =\frac{1}{2} \frac{\mathrm{d}}{\mathrm{d} t}\left(\int_{V} \mathrm{~d}^{3} x T^{00} x^{i} x^{j}\right) \equiv \frac{1}{2} \ddot{I}^{i j}, \\
\int_{V} \mathrm{~d}^{3} x T^{0 i} & =-\frac{\mathrm{d}}{\mathrm{d} t}\left(\int_{V} \mathrm{~d}^{3} x T^{00} x^{i}\right), \\
\int \mathrm{d}^{3} x T^{i j} x^{k} & =\frac{1}{3} \int \mathrm{d}^{3} x\left(T^{i j} x^{k}+T^{k i} x^{j}+T^{j k} x^{i}\right)+\frac{1}{3} \int \mathrm{d}^{3} x\left(2 T^{i j} x^{k}-T^{i k} x^{j}-T^{j k} x^{i}\right), \\
& =\frac{1}{6} \frac{\mathrm{d}^{2}}{\mathrm{~d} t^{2}}\left(\int \mathrm{d}^{3} x T^{00} x^{i} x^{j} x^{k}\right)+\frac{1}{3} \frac{\mathrm{d}}{\mathrm{d} t}\left[\int \mathrm{d}^{3} x\left(T^{0 i} x^{k} x^{j}+T^{0 j} x^{k} x^{i}-2 T^{0 k} x^{i} x^{j}\right)\right] .
\end{aligned}
$$

Hence the electric quadrupole coupling $T^{i j} h_{i j}$ give rise to the $\frac{1}{2} \ddot{Q}^{i j} R_{0 i 0 j}$ term, where at linear order in terms of the Kaluza-Klein fields $\phi, A_{i}, \sigma_{i j}$

$$
R_{0 i 0 j} \simeq \frac{1}{2}\left(\ddot{\sigma}_{i j}-\dot{A}_{i, j}-\dot{A}_{j, i}-2 \phi_{, i j}-2 \delta_{i j} \frac{\ddot{\phi}}{d-2}\right)+O\left(h^{2}\right)
$$

and using

$$
\begin{aligned}
\left(T^{0 i} x^{k} x^{j}-T^{0 k} x^{i} x^{j}\right) \sigma_{i j, k} & =T^{0 m} x^{n} x^{j}\left(\delta_{m}^{l} \delta_{n}^{k}-\delta_{m}^{k} \delta_{n}^{l}\right) \sigma_{l j, k} \\
& =-\epsilon_{i m n} x^{m} T^{0 n} x^{j} \frac{1}{2} \epsilon^{i k l}\left(\sigma_{k j, l}-\sigma_{l j, k}\right)
\end{aligned}
$$

one finds the gravitational magnetic quadrupole coupling eq. (2.5) using the definition of the magnetic part of the Riemann tensor

$$
\mathcal{B}_{i j}=\frac{1}{2} \epsilon_{i k l} R_{0 j k l} \simeq \frac{1}{4 \Lambda} \epsilon_{i k l}\left[\dot{\sigma}_{j k, l}-\dot{\sigma}_{j l, k}+A_{l, j k}-A_{k, j l}+\frac{2}{d-2}\left(\dot{\phi}_{, k} \delta_{j l}-\dot{\phi}_{, l} \delta_{j k}\right)\right] .
$$

Analogously for the axion field one has

$$
\begin{array}{r}
\int \mathrm{d} t\left[\left(\int_{V} \mathrm{~d}^{3} x s^{i j}\right) H_{0 i j}+\left(\int_{V} \mathrm{~d}^{3} x s^{i j} x^{k}\right) H_{0 i j, k}+\left(\int_{V} \mathrm{~d}^{3} x s^{i j} v^{k}\right) H_{i j k}+O\left(v^{2}\right)\right] \\
=\int \mathrm{d} t\left[\left(\int_{V} \mathrm{~d}^{3} x s^{i j}\right) H_{0 i j}+\left(\int_{V} \mathrm{~d}^{3} x s^{i j} x^{k}\right)\left(H_{i j 0, k}-\dot{H}_{i j k}\right)+O\left(v^{2}\right)\right],
\end{array}
$$

where integration by part has been used and terms involving $s^{0 i} \simeq s^{i j} v_{j}$ have been neglected since they enter at order $v^{2}$ with respect to the leading one. Finally introducing the spin density pseudo-vector $\tilde{s}^{i}$ dual to $s^{i j}$ one has for the coupling of the first moment of the spin coupling to the axion

$$
\begin{aligned}
& \frac{1}{2} \epsilon^{i j l} \int_{V}\left(\tilde{s}^{l} x^{k}+\tilde{s}^{k} x^{l}+\tilde{s}^{l} x^{k}-\tilde{s}^{k} x^{l}\right)\left(H_{i j 0, k}-\dot{H}_{i j k}\right) \\
& \quad=\frac{4}{3} J^{k l} \epsilon^{i j l}\left(B_{0 i, k}+\dot{B}_{i k}+B_{k 0, i}\right)_{, j}+\frac{1}{2} \epsilon^{i j l} \int_{V}\left(\tilde{s}^{l} x^{k}-\tilde{s}^{k} x^{l}\right)\left(B_{0 i, k}+\dot{B}_{i k}\right),
\end{aligned}
$$

where it has been used that the leading spin contribution to the magnetic quadrupole is (the traceless part of) $\frac{3}{4}\left(\tilde{s}^{l} x^{k}+\tilde{s}^{k} x^{l}\right)$ [30], thus recovering the magnetic quadrupole coupling to the axion in eq. (2.5), beside a coupling to the antisymmetric first moment of the spin which has no gravitational analog. 


\section{A.2 Graviton, dilaton, axion action up to cubic interaction}

The bulk action is needed for the computations of this paper up to cubic interaction in gravitational, dilatonic and axionic field and it is reported here explictly:

$$
\begin{aligned}
& \mathcal{S}_{\text {bulk }} \supset \int \mathrm{d}^{d+1} x \sqrt{-\gamma}\left\{\frac{1}{4}\left[(\vec{\nabla} \sigma)^{2}-2\left(\vec{\nabla} \sigma_{i j}\right)^{2}-\left(\dot{\sigma}^{2}-2\left(\dot{\sigma}_{i j}\right)^{2}\right) \mathrm{e}^{\frac{-c_{d} \phi}{\Lambda}}\right]-c_{d}\left[(\vec{\nabla} \phi)^{2}-\dot{\phi}^{2} \mathrm{e}^{-\frac{c_{d} \phi}{\Lambda}}\right]\right. \\
& +\left[\frac{F_{i j}^{2}}{2}+(\vec{\nabla} \cdot \vec{A})^{2}-\dot{\vec{A}}^{2} \mathrm{e}^{-\frac{c_{d} \phi}{\Lambda}}\right] \mathrm{e}^{\frac{c_{d} \phi}{\Lambda}}+\frac{2}{\Lambda}\left[\left(F_{i j} A^{i} \dot{A^{j}}+\vec{A} \cdot \dot{\vec{A}}(\vec{\nabla} \cdot \vec{A})\right)-c_{d} \dot{\phi} \vec{A} \cdot \vec{\nabla} \phi\right] \\
& -\frac{1}{\Lambda}\left(\frac{\sigma}{2} \delta^{i j}-\sigma^{i j}\right)\left(\sigma_{i k}{ }^{l} \sigma_{j l}{ }^{, k}-\sigma_{i k}{ }^{, k} \sigma_{j l}{ }^{l}+\sigma_{, i} \sigma_{j k}, k-\sigma_{i k, j} \sigma^{, k}\right) \\
& +\frac{\dot{\sigma}_{i j}}{\Lambda}\left(-\delta^{i j} A_{l} \hat{\Gamma}_{k k}^{l}+2 A_{k} \hat{\Gamma}_{i j}^{k}-2 A^{i} \hat{\Gamma}_{k k}^{j}\right) \\
& +2(d-1)\left[e^{-c_{d} \frac{\phi}{\Lambda}} \dot{\psi}^{2}-(\nabla \psi)^{2}-\frac{2}{\Lambda} \dot{\psi} \vec{A} \cdot \vec{\nabla} \psi\right] \\
& \left.-\frac{1}{6} e^{\frac{4 \psi}{\Lambda}+2\left(c_{d}-2\right) \frac{\phi}{\Lambda}}\left(H_{i j k}^{2}-3 e^{-c_{d} \frac{\phi}{\Lambda}} H_{i j 0}^{2}\right)-A^{i} H_{i j k} H_{0 j k}+\frac{1}{2} H_{i j k} H_{i j l} \sigma^{k l}\right\} .
\end{aligned}
$$

Contractions between explicit space (Latin) indices are done with flat metric, when indices are understood contractions are made including the field $\sigma_{i j}$ in the metric, e.g. $\vec{A} \cdot \nabla \phi=A_{i} \phi_{, j}\left(\delta^{i j}-\sigma^{i j}+\ldots\right)$.

\section{B Vanishing integrals}

To recast the gravitational amplitudes in eqs. (4.3) and (4.8) into a double-copy structure, we now show that the pieces that do not fit the factorizable form vanish identically. Performing the change of integration variables $\mathbf{q} \rightarrow-\left(\mathbf{k}+\mathbf{k}^{\prime}\right)$ one immediately finds:

$$
\begin{aligned}
& \int_{\mathbf{k}, \mathbf{q}} \frac{k_{i_{1}} \cdots k_{i_{n}}(k+q)_{k_{1}} \cdots(k+q)_{k_{n}}}{\left(\mathbf{k}^{2}-k_{0}^{2}\right)\left((\mathbf{k}+\mathbf{q})^{2}-k_{0}^{2}\right) \mathbf{q}^{2}}\left(q_{i} k_{k}+q_{k}(k+q)_{i}\right) \\
& \quad=(-1)^{n} \int_{\mathbf{k}, \mathbf{k}^{\prime}} \frac{k_{i_{1}} \cdots k_{i_{n}} k_{k_{1}}^{\prime} \cdots k_{k_{n}}^{\prime}}{\left(\mathbf{k}^{2}-k_{0}^{2}\right)\left(\mathbf{k}^{\prime 2}-k_{0}^{2}\right)\left(\mathbf{k}+\mathbf{k}^{\prime}\right)^{2}}\left[k_{i}^{\prime} k_{k}^{\prime}-k_{i} k_{k}\right],
\end{aligned}
$$

which vanishes when contracted with $\delta_{j l} I^{i j i_{1} \cdots i_{n}}\left(k_{0}\right) I^{k l k_{1} \cdots k_{n}}\left(-k_{0}\right)$, being antisymmetric under swapping $k \leftrightarrow k^{\prime}$.

Terms proportional to gravitational radiation propagators $\left(\mathbf{k}^{2}-k_{0}\right)$ or $\left[(\mathbf{k}+\mathbf{q})^{2}-k_{0}^{2}\right]$ also vanish identically, given that

$$
\int_{\mathbf{q}} \frac{q_{i_{1}} \cdots q_{i_{n}}}{\mathbf{q}^{2}}=0
$$

for any $n$. 
For terms proportional to $\mathbf{q}^{2}$ we observe that

$$
\int_{\mathbf{k}, \mathbf{k}^{\prime}} \frac{k_{i_{1}} \cdots k_{i_{2 n}} k_{k_{1}}^{\prime} \cdots k_{k_{2 m}}^{\prime}}{\left(\mathbf{k}^{2}-k_{0}^{2}\right)\left(\mathbf{k}^{\prime 2}-k_{0}^{2}\right)} \propto \delta^{\left(i_{1} i_{2}\right.} \cdots \delta^{\left.i_{2 n-1} i_{2 n}\right)} \delta^{\left(k_{1} k_{2}\right.} \cdots \delta^{\left.k_{2 m-1} k_{2 m}\right)}
$$

vanish when contracted with traceless tensors, and also trivially vanish when an odd number of momentum factors in the numerator is involved.

Finally by rearranging the last term as follows

$$
\begin{aligned}
\int_{\mathbf{k}, \mathbf{q}} & \frac{k_{i_{1}} \cdots k_{i_{n}}(k+q)_{k_{1}} \cdots(k+q)_{k_{n}}}{\left(\mathbf{k}^{2}-k_{0}^{2}\right)\left((\mathbf{k}+\mathbf{q})^{2}-k_{0}^{2}\right) \mathbf{q}^{2}} \times\left(k_{i} k_{j} q_{k} q_{l}-q_{i} q_{j}(k+q)_{k}(k+q)_{l}\right) \\
= & (-1)^{n} \int_{\mathbf{k}, \mathbf{k}^{\prime}} \frac{k_{i_{1}} \cdots k_{i_{n}} k_{k_{1}}^{\prime} \cdots k_{k_{n}}^{\prime}}{\left(\mathbf{k}^{2}-k_{0}^{2}\right)\left(\mathbf{k}^{\prime 2}-k_{0}^{2}\right)\left(\mathbf{k}+\mathbf{k}^{\prime}\right)^{2}} \\
& \times\left[k_{i} k_{j} k_{k} k_{l}-k_{i}^{\prime} k_{j}^{\prime} k_{k}^{\prime} k_{l}^{\prime}+k_{i} k_{l}^{\prime}\left(k_{j} k_{k}-k_{j}^{\prime} k_{k}^{\prime}\right)+k_{j} k_{k}^{\prime}\left(k_{i} k_{l}-k_{i}^{\prime} k_{l}^{\prime}\right)\right],
\end{aligned}
$$

one sees that it vanishes when contracted with $I^{i j i_{1} \cdots i_{n}} I^{k l k_{1} \cdots k_{n}}$ because of the anti-symmetry under $k \leftrightarrow k^{\prime}$. This concludes the demonstration that terms in eqs. (4.3) and (4.8) that do not fit in the double copy structure vanish. Last line in eq. (4.12) can be shown to vanish with the same reasoning, using momenta $\mathbf{k}$ and $\mathbf{k}^{\prime} \equiv-(\mathbf{k}+\mathbf{q})$.

Open Access. This article is distributed under the terms of the Creative Commons Attribution License (CC-BY 4.0), which permits any use, distribution and reproduction in any medium, provided the original author(s) and source are credited.

\section{References}

[1] H. Kawai, D.C. Lewellen and S.H.H. Tye, A Relation Between Tree Amplitudes of Closed and Open Strings, Nucl. Phys. B 269 (1986) 1 [INSPIRE].

[2] N.E.J. Bjerrum-Bohr, String theory and the mapping of gravity into gauge theory, Phys. Lett. B 560 (2003) 98 [hep-th/0302131] [INSPIRE].

[3] Z. Bern, J.J.M. Carrasco and H. Johansson, New Relations for Gauge-Theory Amplitudes, Phys. Rev. D 78 (2008) 085011 [arXiv:0805.3993] [inSPIRE].

[4] J.J.M. Carrasco, Gauge and Gravity Amplitude Relations, in proceedings of the Theoretical Advanced Study Institute in Elementary Particle Physics: Journeys Through the Precision Frontier: Amplitudes for Colliders, Boulder, CO, U.S.A., 2-27 June 2014, L. Dixon and F. Petriello eds., World Scientific (2015), pp. 477-557 [arXiv: 1506. 00974] [INSPIRE].

[5] Z. Bern, J.J. Carrasco, M. Chiodaroli, H. Johansson and R. Roiban, The Duality Between Color and Kinematics and its Applications, arXiv:1909.01358 [INSPIRE].

[6] R. Monteiro, D. O'Connell and C.D. White, Black holes and the double copy, JHEP 12 (2014) 056 [arXiv: 1410.0239] [inSPIRE].

[7] K. Kim, K. Lee, R. Monteiro, I. Nicholson and D. Peinador Veiga, The Classical Double Copy of a Point Charge, JHEP 02 (2020) 046 [arXiv: 1912.02177] [INSPIRE]. 
[8] W.D. Goldberger and A.K. Ridgway, Radiation and the classical double copy for color charges, Phys. Rev. D 95 (2017) 125010 [arXiv:1611.03493] [InSPIRE].

[9] W.D. Goldberger, J. Li and S.G. Prabhu, Spinning particles, axion radiation, and the classical double copy, Phys. Rev. D 97 (2018) 105018 [arXiv:1712.09250] [INSPIRE].

[10] J. Li and S.G. Prabhu, Gravitational radiation from the classical spinning double copy, Phys. Rev. D 97 (2018) 105019 [arXiv: 1803.02405] [inSPIRE].

[11] C.-H. Shen, Gravitational Radiation from Color-Kinematics Duality, JHEP 11 (2018) 162 [arXiv: 1806. 07388] [INSPIRE].

[12] W.D. Goldberger and J. Li, Strings, extended objects, and the classical double copy, JHEP 02 (2020) 092 [arXiv: 1912.01650] [INSPIRE].

[13] Y.F. Bautista and A. Guevara, From Scattering Amplitudes to Classical Physics: Universality, Double Copy and Soft Theorems, arXiv:1903.12419 [INSPIRE].

[14] J. Plefka, J. Steinhoff and W. Wormsbecher, Effective action of dilaton gravity as the classical double copy of Yang-Mills theory, Phys. Rev. D 99 (2019) 024021 [arXiv: 1807.09859] [INSPIRE].

[15] J. Plefka, C. Shi, J. Steinhoff and T. Wang, Breakdown of the classical double copy for the effective action of dilaton-gravity at NNLO, Phys. Rev. D 100 (2019) 086006 [arXiv: 1906.05875] [INSPIRE].

[16] Z. Bern, C. Cheung, R. Roiban, C.-H. Shen, M.P. Solon and M. Zeng, Scattering Amplitudes and the Conservative Hamiltonian for Binary Systems at Third Post-Minkowskian Order, Phys. Rev. Lett. 122 (2019) 201603 [arXiv: 1901.04424] [INSPIRE].

[17] Z. Bern, C. Cheung, R. Roiban, C.-H. Shen, M.P. Solon and M. Zeng, Black Hole Binary Dynamics from the Double Copy and Effective Theory, JHEP 10 (2019) 206 [arXiv: 1908.01493] [INSPIRE].

[18] L. Blanchet, Gravitational Radiation from Post-Newtonian Sources and Inspiralling Compact Binaries, Living Rev. Rel. 17 (2014) 2 [arXiv:1310.1528] [INSPIRE].

[19] S. Foffa and R. Sturani, Conservative dynamics of binary systems to fourth Post-Newtonian order in the EFT approach I: Regularized Lagrangian, Phys. Rev. D 100 (2019) 024047 [arXiv: 1903.05113] [INSPIRE].

[20] S. Foffa, R.A. Porto, I. Rothstein and R. Sturani, Conservative dynamics of binary systems to fourth Post-Newtonian order in the EFT approach II: Renormalized Lagrangian, Phys. Rev. D 100 (2019) 024048 [arXiv: 1903.05118] [InSPIRE].

[21] W.D. Goldberger and I.Z. Rothstein, An Effective field theory of gravity for extended objects, Phys. Rev. D 73 (2006) 104029 [hep-th/0409156] [INSPIRE].

[22] A.J. Hanson and T. Regge, The Relativistic Spherical Top, Annals Phys. 87 (1974) 498 [INSPIRE].

[23] J. Scherk and J.H. Schwarz, Dual Models and the Geometry of Space-Time, Phys. Lett. B 52 (1974) 347 [INSPIRE].

[24] Z. Bern and A.K. Grant, Perturbative gravity from QCD amplitudes, Phys. Lett. B 457 (1999) 23 [hep-th/9904026] [INSPIRE].

[25] B. Kol and M. Smolkin, Non-Relativistic Gravitation: From Newton to Einstein and Back, Class. Quant. Grav. 25 (2008) 145011 [arXiv:0712.4116] [INSPIRE]. 
[26] S. Foffa and R. Sturani, Hereditary terms at next-to-leading order in two-body gravitational dynamics, Phys. Rev. D 101 (2020) 064033 [arXiv:1907.02869] [INSPIRE].

[27] L. Blanchet and T. Damour, Tail Transported Temporal Correlations in the Dynamics of a Gravitating System, Phys. Rev. D 37 (1988) 1410 [InSPIRE].

[28] S. Foffa and R. Sturani, Tail terms in gravitational radiation reaction via effective field theory, Phys. Rev. D 87 (2013) 044056 [arXiv:1111.5488] [INSPIRE].

[29] W.D. Goldberger and A.K. Ridgway, Bound states and the classical double copy, Phys. Rev. D 97 (2018) 085019 [arXiv: 1711.09493] [INSPIRE].

[30] L. Blanchet, A. Buonanno and G. Faye, Higher-order spin effects in the dynamics of compact binaries. II. Radiation field, Phys. Rev. D 74 (2006) 104034 [Erratum ibid. 75 (2007) 049903] [Erratum ibid. 81 (2010) 089901] [gr-qc/0605140] [INSPIRE]. 